\title{
Keeping tabs on the mabs: a hematopathologist's dilemma
}

\author{
William R. Macon ${ }^{1}$
}

Published online: 22 February 2019

(C) Springer-Verlag GmbH Germany, part of Springer Nature 2019

Rituximab, a chimeric monoclonal antibody (mab) directed against the CD20 antigen expressed on the cell membrane of benign and most neoplastic mature B cells, was the first mab to achieve regulatory approval for human use for the treatment of relapsed/refractory indolent non-Hodgkin lymphomas by the US Food and Drug Administration (FDA) in 1997 and by the European Medicines Agency in 1998 [1]. This effectively ushered in the era of immunotherapy, immunochemotherapy, and immunoconjugate therapy for more targeted treatment of a variety of malignancies.

Since then, there has been a near tidal wave of new mabs for use as monotherapeutic agents or in combination therapy for many hematolymphoid neoplasms. One such agent is brentuximab vedotin, a chimeric mab that targets the CD30 antigen, which has had FDA approval for treating patients with relapsed classic Hodgkin lymphoma (cHL) or systemic anaplastic large cell lymphoma (ALCL), with previously untreated stage III or IV cHL, and with refractory primary cutaneous ALCL or CD30positive mycosis fungoides. Brentuximab has recently been granted FDA approval for first-line combined immunochemotherapy in patients diagnosed with systemic ALCL and other CD30-positive peripheral T cell lymphomas (PTCLs) based on the results of the ECHELON-2 clinical trial [2]. The authors of this study concluded that their protocol of brentuximab vedotin in combination with cyclophosphamide, doxorubicin, and prednisone $(\mathrm{A}+$ CHP) has the potential "to become a new standard of care for many patients with CD30-positive peripheral T-cell lymphoma" [2]. Others have hailed it similarly, such as Dr. Vincent DeVita from the Yale School of Public Health, New Haven, CT, USA: "I think the authors are correct in stating this study supports A+CHP as the new standard of care for this troublesome subset of non-

William R. Macon

Macon.William@mayo.edu

1 Mayo Clinic, Rochester, MN, USA
Hodgkin lymphoma" [3] and Dr. Mary Gleeson from Guys' and St Thomas' NHS Foundation Trust, London, UK: "The findings of the ECHELON-2 trial undoubtedly represent a major and practice-changing step forward in the treatment of patients with CD30-positive peripheral T-cell lymphoma" [4].

This is also a practice-changer for hematopathologists as CD30 immunohistochemical staining is now needed for all forms of PTCL. However, the trial and the FDA have left unanswered questions for those of us on the diagnostic side of the fence. The methodology for ECHELON-2, in the paper by Horwitz and associates and as further specified in its supplementary appendix, had three criteria for inclusion as CD30positive disease by local pathologists on paraffin-embedded tissue blocks or unstained slides from diagnostic core or excisional/incisional biopsies using immunohistochemistry and the BerH2 antibody: $\mathrm{CD} 30$ detected in $\geq 10 \%$ of neoplastic cells (or "in cases where enumeration of neoplastic cells was not possible, total lymphocytes may have been used"); CD30 staining of any intensity above background; and CD30 staining in membranous, cytoplasmic, and/or Golgi pattern [2]. Left unspecified were many issues relevant to hematopathologists, including both pre-analytic (e.g., type of fixative used, fixation-time, time interval from cutting of slides to point of staining for submitted unstained slides, etc.) and analytic (e.g., immunohistochemical methodology employed) concerns. The FDA made no specifications regarding what is considered CD30-positive, such that any level of CD30 positivity may be regarded as allowable for treatment by some hematologists/oncologists (Dr. Stephen M. Ansell, Division of Hematology, Mayo Clinic, Rochester, MN, USA, personal communication).

While ECHELON-2 leaves some cumbersome concerns for hematopathologists, greater clarity in test methodology will be needed in the future as more and more mabs are being developed and utilized as part of innovative but very expensive treatment protocols, such as chimeric antigen receptor (CAR) T cell therapy. It is likely that private and government payers will want less guesswork in the laboratory testing needed for patient eligibility. 
William R. Macon, MD

Editor-in-Chief

Journal of Hematopathology

Publisher's note Springer Nature remains neutral with regard to jurisdictional claims in published maps and institutional affiliations.

\section{References}

1. Salles G, Barrett M, Foà R, Maurer J, O'Brien S, Valente N, Wenger M, Maloney DG (2017) Rituximab in B-cell hematologic malignancies: a review of 20 years of clinical experience. Adv Ther 34:2232-2273
2. Horwitz S, O'Connor OA, Pro B, Illidge T, Fanale M, Advani R, Bartlett NL, Christensen JH, Morschhauser F, Domingo-Domenech E, Rossi G, Kim WS, Feldman T, Lennard A, Belada D, Illès A, Tobinai K, Tsukasaki K, Yeh S-P, Shustov A, Hüttman A, Savage KJ, Yuen S, Iyer S, Zinzani PL, Hua Z, Little M, Rao S, Woolery J (2019) Brentuximab vedotin with chemotherapy for CD30-positive peripheral T-cell lymphoma (ECHELON-2): a global, double-blind, randomised, phase 3 trial. Lancet 393:229-240

3. Douglas D (2019) Brentuximab vedotin and chemotherapy effective in peripheral T-cell lymphoma. Oncology Newswatch

4. Gleeson M (2019) Moving to a higher echelon in CD30-positive Tcell lymphoma. Lancet 393:201-202 